\title{
Characterization of Unlabeled Radial Level Planar Graphs (Extended Abstract)
}

\author{
J. Joseph Fowler \\ Department of Computer Science, University of Arizona \\ jfowler@cs.arizona.edu
}

\begin{abstract}
Suppose that an $n$-vertex graph has a distinct labeling with the integers $\{1, \ldots, n\}$. Such a graph is radial level planar if it admits a crossings-free drawing under two constraints. First, each vertex lies on a concentric circle such that the radius of the circle equals the label of the vertex. Second, each edge is drawn with a radially monotone curve. We characterize the set of unlabeled radial level planar (URLP) graphs that are radial level planar in terms of 7 and 15 forbidden subdivisions depending on whether the graph is disconnected or connected, respectively. We also provide linear-time drawing algorithms for any URLP graph.
\end{abstract}

\section{Introduction}

Visualizing social networks with respect to centrality, the relative importance that actors hold within a relational structure, yields a graphical representation that conveys domain-specific hierarchical information that aids in policy network analysis [2]. When possible, planar layouts are preferred where actors are placed at distances from the origin based upon their level of importance. Thus, vertices are constrained to lie along concentric circles, called rings, with radii proportional to the centrality of the respective actors. Radially monotone curves can be used for edges, which denote relationships between actors. Each curve lies between the rings of the endpoints of the edge. Such layouts are radial level planar if the drawing is crossings free. If straight-line edges are used, edges may cross rings interior to their endpoints, which can decrease readability; see Fig. 1.

Spiral edges have the added advantage that the radii used for the rings can vary uniformly. This is not the case with straight-line edges. For instance, an outerplanar graph admits a straight-line planar drawing in which the vertices lie on a unit circle. If each vertex is perturbed to lie on a circle of different radii, planarity may be lost unless extremely minute perturbations are used [4].

Some social networks have a structure that are conducive to the dynamic visualization of actors whose centrality changes over time. The most dynamic networks are radial level planar regardless of the centrality of the actors. In representing a social network, we take the corresponding graph and label each vertex with an integer denoting its degree of importance. This then becomes the distance that each actor is placed from the origin. 


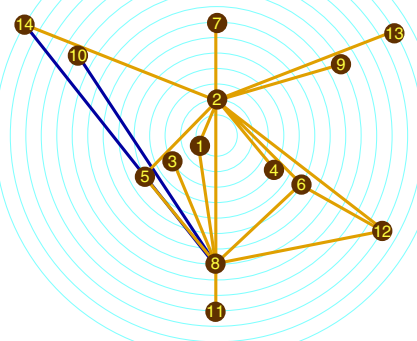

(a) Straight-line edges

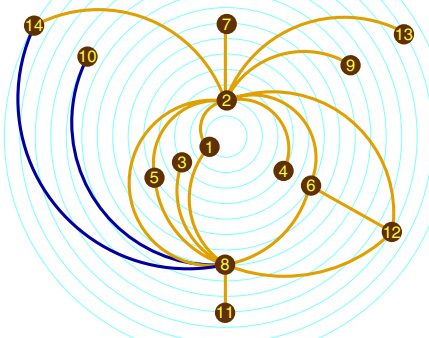

(b) Circular arc edges

Fig. 1. Drawing a radial graph with different edge types

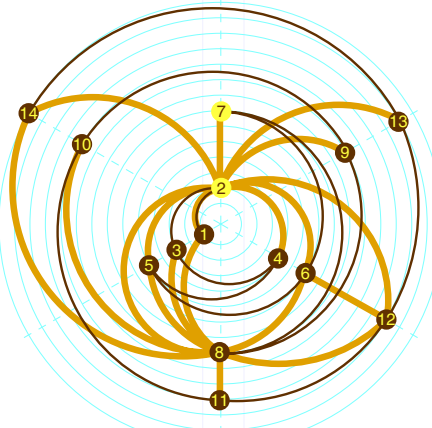

(a) Radial

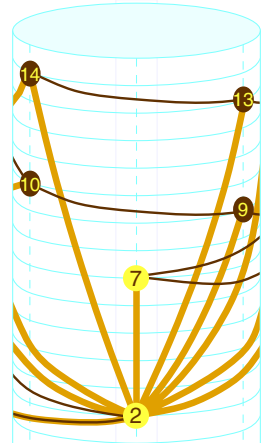

(b) Cylindrical

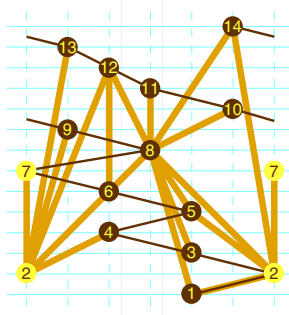

(c) Horizontal

Fig. 2. Three equivalent representations of radial level graphs

This leads us to consider the set of unlabeled radial level planar (URLP) graphs that have a radial level planar layout for any distinct labeling. The term "unlabeled" implies that the underlying structure of the graph determines whether radial level planarity is always possible, independent of any labeling scheme. Alternatively, URLP graphs can be defined as the set of graphs that can always be drawn simultaneously without crossings with a radially monotone path. Labels are given by the order that the vertices occur along the path; see Fig. 2(a). This is related to the problem of simultaneous embedding in which multiple planar graphs are drawn simultaneously on the same vertex set $[3$.

As an equivalent graphical representation, a radial level graph can be drawn on a cylinder in which the rings are circles of equal radii at different heights along the cylinder; see Fig. 2(b). The radial drawing may wrap around the cylinder. A third alternate representation comes from cutting the cylinder along a vertical line (a ray from the origin in Fig. 2(a)) and flattening the cut surface onto a plane. The rings then become horizontal lines, and the edges becomes straightlines that can "wrap" from the right to the left side of the drawing; see Fig. 2(c). 
If no edges wrap in this third representation, then the graph is level planar. Analogous to URLP graphs, graphs that are level planar for any distinct labeling are unlabeled level planar (ULP). We use this last representation when drawing radial graphs given its compactness and relationship to ULP graphs.

\subsection{Related Previous Work}

Radial level planar graphs can be recognized and embedded in linear time 1. A linear-time straight-line drawing algorithm exists for level planar graphs [511,

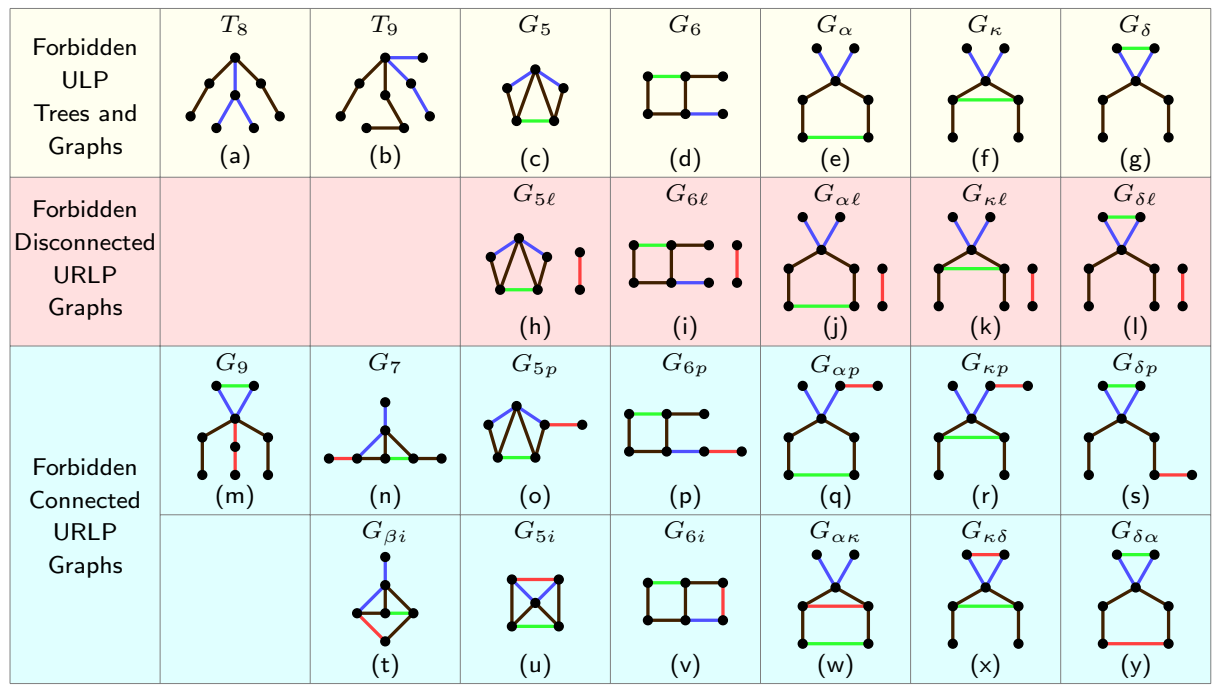

Fig. 3. Forbidden ULP and URLP trees and graphs

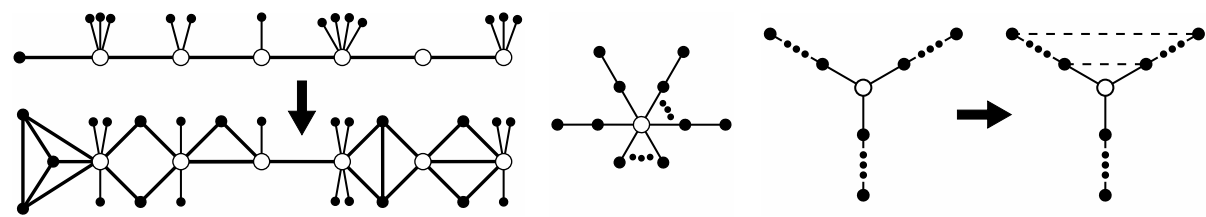

(a) caterpillar $\rightarrow$ generalized caterpillar

(b) radius-2 star

(c) degree-3 spider $\rightarrow$ extended 3-spider

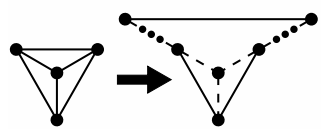

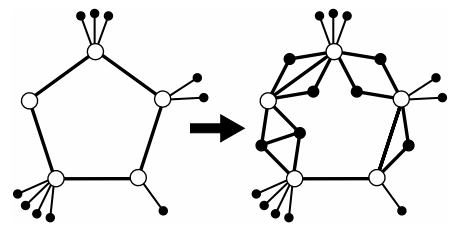

(e) circular caterpillar $\rightarrow$ generalized circular caterpillar

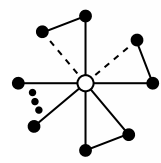

(f) tri- $K_{3}$ star

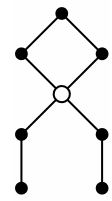

(g) $G_{8}$

Fig. 4. Classes of ULP trees and graphs in (a)-(d) and classes of additional URLP graphs in $(\mathrm{e})-(\mathrm{g})$ where dashed edges are optional and white vertices are cut vertices 


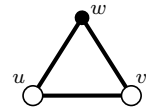

(a) $K_{3}$ block

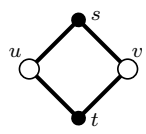

(b) $C_{4}$ block

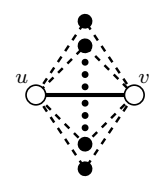

$\left(K_{3}\right)^{*}$
block

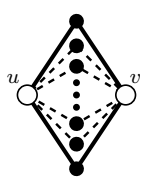

(d) $\left(C_{4}\right)^{+}$ block

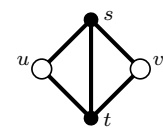

(e) diamond block

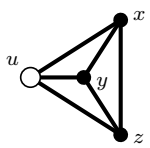

(f) $K_{4}$ block

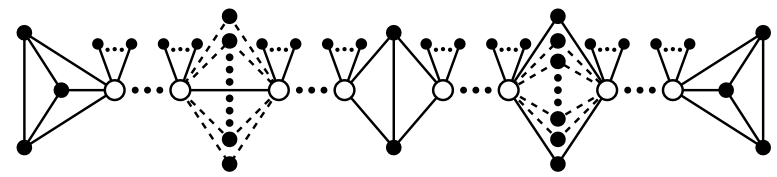

(g) generalized caterpillar

Fig. 5. Six types of ULP blocks where the joining blocks in (a)-(e) can be substituted for an internal edge $(u, v)$ of a caterpillar and the ending block in (f) can be substituted for at most one leaf edge $(u, \ell)$ incident to each endpoint $u$ of the spine in order to form $(\mathrm{g})$

which can be adapted for the radial case in terms of radially monotone curves. These algorithms are for a given labeling in which duplicate labels are permitted.

ULP trees have been characterized in terms of the two forbidden trees $T_{8}$ and $T_{9}$ in Fig. 3 (a)-(b) 6 6]. As a result, the universe of trees is partitioned into those that contain a subtree homeomorphic to $T_{8}$ or $T_{9}$ and the class of ULP trees. An ULP tree was shown to be either (i) a caterpillar (a tree where the removal of all leaves yields a path, its spine), (ii) a radius-2 star (a subdivided $K_{1, k}$ such that $k \geq 3$ where one or more edges have been subdivided exactly once), or (iii) a degree-3 spider (an arbitrarily subdivided $K_{1,3}$ ); see Fig. 4(a)-(c).

Similarly, ULP graphs have been characterized in terms of the seven forbidden graphs in Fig. 3) (a)-(g) 910 where any graph without a subdivision of one of these seven forbidden graphs is ULP. The class of ULP graphs consists of (i) generalized caterpillars (GCs) (formed by substituting edges of a caterpillar for ULP blocks as described in Fig. 5), (ii) rstars (R2Ss), (iii) extended 3-spiders (E3Ss) (formed by adding two optional edges to a degree-3 spider so as to connect two leaf vertices or two neighbors of the root vertex of degree 3 ), or (iv) extended $K_{4}$ subgraphs (EK4s) (a connected subgraph of a subdivided $K_{4}$ where exactly one edge has been arbitrarily subdivided); see Fig. 4(a)-(d). All ULP graphs have level planar drawings that only require linear time and space; see Fig. 6 .

\subsection{Our Contribution}

Of the four classes of ULP graphs, only generalized caterpillars are drawn blockby-block proceeding left to right as in Fig. 6(a). This is unlike extended 3-spiders that can have spirals when drawn as in Fig. 6(c). Given that edges can wrap around in a radial level planar graph, this leads to the URLP class of generalized circular caterpillars (GCCs). These are constructed by substituting any of the joining ULP blocks in Fig. 5(a)-(e) for a cycle edge of a circular caterpillar (a graph where the removal of all endpoints yields a cycle); see Fig. 4(e). Observe that extended $K_{4}$ subgraphs are a subclass of generalized (circular) caterpillars. 

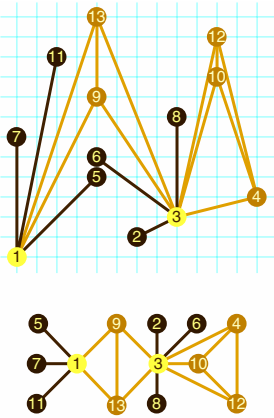

(a) generalized caterpillar

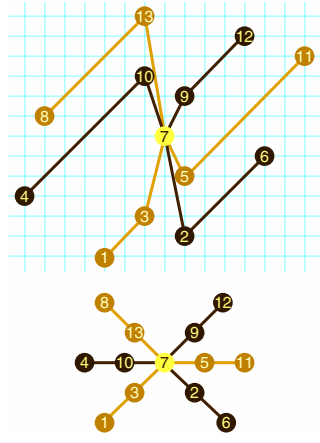

(b) radius-2 star
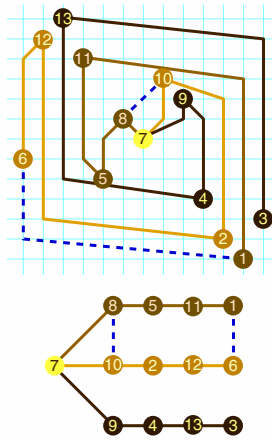

(c) extended 3-spider
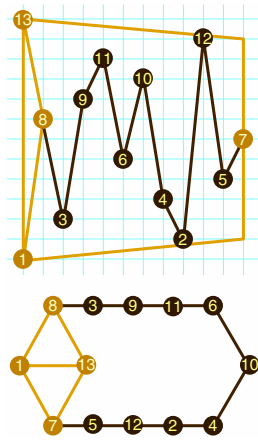

(d) extended $K_{4}$

Fig. 6. Level planar drawings of the four classes of ULP graphs

A $t r i-K_{3}$ star (TK3S) is formed by adding up to three edges to a radius- 2 star (with at most three subdivided edges) that connect one, two, or three leaves to the root vertex as in Fig. 4(f). The graph $G_{8}$ in Fig. 4(g) is a subdivision of $G_{\delta}$ in Fig. 3(g), a tri- $K_{3}$ star, where the 3 -cycle has been subdivided to form a 4-cycle. Both classes of graphs admit radial level planar drawings where an edge of each cycle can wrap around so as to avoid a crossing.

We extend the ULP characterization to all URLP graphs as follows:

1. First, we show that a disconnected graph is URLP if and only if it neither contains a subgraph homeomorphic to either tree in Fig. 3(a) or (b) nor to one of the disconnected graphs in Fig. 3(h)-(l) for a total of 7 forbidden subdivisions. We prove that this is equivalent to each component being ULP.

2. Second, we show that a connected graph is URLP if and only if it neither contains a subgraph homeomorphic to either of the trees in Fig. 33(a)-(b) nor to one of the connected graphs in Fig. 3 $(\mathrm{m})-(\mathrm{y})$ for a total of 15 forbidden subdivisions. We prove that this is equivalent to the graph either being ULP or belonging to one of the three additional classes of URLP connected graphs, which are generalized circular caterpillars, tri- $K_{3}$ stars, and graphs isomorphic to $G_{8}$.

3. Third, we provide $O(n)$-time drawing algorithms for each new class of $n$-vertex URLP graphs that are drawn on $O(n) \times n$ integer grids.

\section{Preliminaries}

Track $\ell_{j}$ is the horizontal line $\{(x, j): x \in \mathbb{R}\}$. Ring $c_{j}$ is the circle $\{(x, y)$ : $\left.x^{2}+y^{2}=j^{2},(x, y) \in \mathbb{R}^{2}\right\}$ in Cartesian coordinates or $\{(j, \theta): j \in \mathbb{R}, 0 \leq \theta \leq 2 \pi\}$ in radial coordinates. Curve $L=\left\{(x(s), y(s)): s_{1} \leq s \leq s_{2}\right\}=\{(r(s), \theta(s))$ : $\left.s_{1} \leq s \leq s_{2}\right\}$ in parametrized Cartesian and radial coordinates, respectively, is $y$-monotone if $y(s)<y\left(s^{\prime}\right)$ when $s<s^{\prime}$ and $r$-monotone if $r(s)<r\left(s^{\prime}\right)$ when $s<s^{\prime}$. A $m \times n$ circular grid consists of $n$ concentric rings $\left\{\left(r^{\prime}, \theta\right): 0 \leq \theta \leq 2 \pi\right\}$ for $r^{\prime} \in\{1, \ldots, n\}$ and $m$ rays $\left\{\left(r, \theta^{\prime}\right): r \in \mathbb{R}\right\}$ for $\theta^{\prime} \in\left\{\frac{2 k \pi}{m}: k \in\{1, \ldots, m\}\right\}$. 
Let $G(V, E)$ be an undirected graph with vertex set $V$ and edge set $E=$ $\{(u, v): u, v \in V, u \neq v\}$ with no isolated vertices. Let $\phi$ be a labeling on $V$ with the integers 1 to $k$, i.e. $\phi: V \mapsto\{1, \ldots, k\}$ where $k \leq|V|$. A level is a set of vertices with the same label where the $j^{\text {th }}$ level is the vertex subset $V_{j}=\{v \in V: \phi(v)=j\}$. If $\phi(u) \neq \phi(v)$ for any $(u, v) \in E$, then $G(V, E, \phi)$ forms a (radial) level graph on $k$ levels. Radial level graph $G(V, E, \phi)$ is (radial) level planar if a drawing can be realized such that (i) each vertex of $V_{j}$ is placed along track $\ell_{j}$ (or ring $c_{j}$ ), (ii) each edge $(u, v)$ is drawn with a $y$-monotone (or $r$-monotone) curve (that can wrap), and (iii) each pair of curves can only intersect at their endpoints. Graph $G(V, E)$ is unlabeled (radial) level planar if (radial) level graph $G(V, E, \phi)$ is (radial) level planar for every bijective labeling $\phi$. A (radial) level planar drawing is also called a realization.

\section{Drawing Unlabeled Radial Level Planar Graphs}

Any graph that has a level planar drawing has a radial level planar realization as seen by the equivalence of the layouts in Fig. 2. Hence, the drawing algorithms for ULP graphs given in [7910] naturally extend to the radial setting. The drawing algorithm for generalized caterpillars detailed in 910 draws each ULP block proceeding left to right; see Fig. 6(a). Extending this algorithm to draw each ULP block of a generalized circular caterpillar proceeding clockwise in a radial setting gives the next lemma. See 8 for a more detailed proof.

Lemma 1. An n-vertex generalized (circular) caterpillar with $m$ ULP blocks can be realized on a $4 m \times n$ (circular) grid in $O(n)$ time for any distinct labeling.

We can also directly extend the drawing algorithms from 7/910] for radius-2 stars and extended 3-spiders to give the next two lemmas, respectively.

Lemma 2. An n-vertex radius-2 star can be realized in $O(n)$ time on a (circular) $(2 n+1) \times n$ grid for any distinct labeling.

Lemma 3. An n-vertex extended 3-spider can be realized in $O(n)$ time on an $(n+1) \times n$ (circular) grid for any distinct labeling.

Next, we show how to realize the remaining two classes of URLP graphs in the subsequent two lemmas; the full proofs of each can be found in [8].

Lemma 4. An n-vertex tri- $K_{3}$ star $G$ can be realized on a $5 \times n$ circular grid in $O(n)$ time for any distinct labeling.

Proof Sketch: Figure 7 illustrates how to handle the four distinct cases of drawing a tri- $K_{3}$ star with the three 3-cycles $r-s-t-r, r-v-w-r$, and $r-x-y-r$ where $\phi(s)>\phi(t), \phi(v)>\phi(w), \phi(x)>\phi(y)$, and $\phi(s)>\phi(v)>\phi(x)$.

Initially, $s, t, x$, and $y$ are placed two units to the right of $r$, while $v, w$ are placed to two units to the left of $r$. In the first case $\phi(t)>\phi(x)$ so that no crossings will occur. In the remaining three cases where $\phi(x)>\phi(t)$, the vertices $t, w$, and $y$ have the minimum labels of any 3-cycle, respectively, and vertices switch sides as depicted in Fig. (7(b)-(d). Finally, leaf edges are either drawn one unit to the left or to the right of $r$ so as to avoid any edge overlap. 


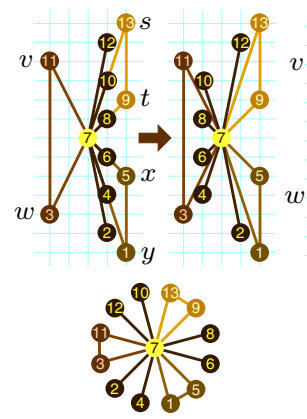

(a) Case 1

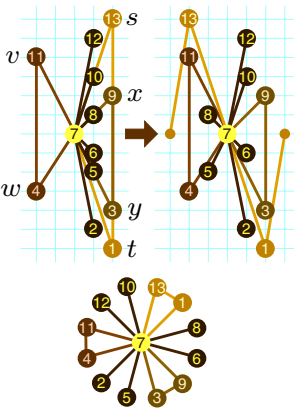

(b) Case 2

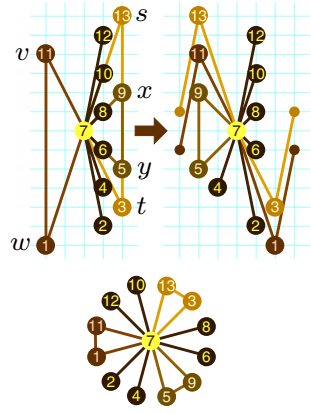

(c) Case 3
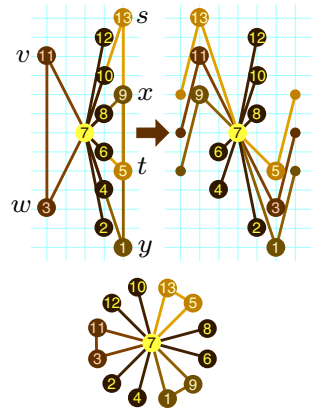

(d) Case 4

Fig. 7. Four cases of how to avoid edge overlaps for tri- $K_{3}$ stars

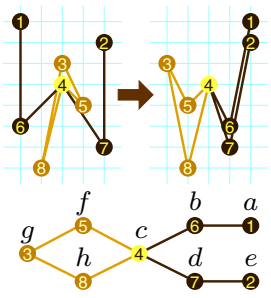

(a) Case 1
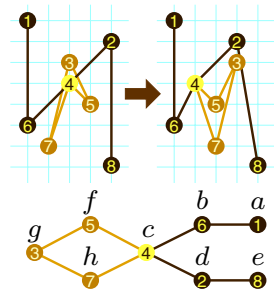

(b) Case 2
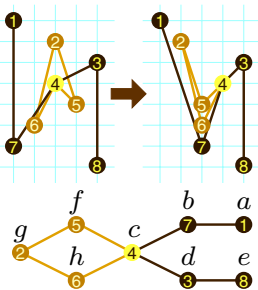

(c) Case 3

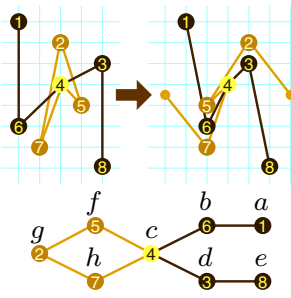

(d) Case 4

Fig. 8. Four cases of how to avoid edge overlaps for $G_{8}$

Lemma 5. The graph $G_{8}$ can be realized on a $5 \times 8$ circular grid in $O(1)$ time for any distinct labeling.

Proof Sketch: If any chain of length 2 of $G_{8}$ is radially monotone, then drawing $G_{8}$ is equivalent to drawing the generalized caterpillar or tri- $K_{3}$ star obtained by replacing the chain with a single edge where either the algorithms of Lemmas 1 or 4 can be used. Otherwise, Fig. 8 depicts the four remaining cases of how to draw $G_{8}$ if this is not the case.

\section{Forbidden Unlabeled Radial Level Planar Graphs}

We define $\mathcal{F}_{\text {ULP }}:=\left\{T_{8}, T_{9}, G_{5}, G_{6}, G_{\alpha}, G_{\kappa}, G_{\delta}\right\}$ in Fig. $3(\mathrm{a})-(\mathrm{g}), \mathcal{T}_{\text {URLP }}:=\left\{T_{8}, T_{9}\right\}$ (forbidden URLP trees) in Fig. 3(a)-(b), D URLP $:=\left\{G_{5 \ell}, G_{6 \ell}, G_{\alpha \ell}, G_{\kappa \ell}, G_{\delta \ell}\right\}$ (disconnected forbidden URLP graphs) in Fig. 33(h)-(l), $\mathcal{C}_{\text {URLP }}:=\left\{G_{9}, G_{7}, G_{5 p}\right.$, $\left.G_{6 p}, G_{\alpha p}, G_{\kappa p}, G_{\delta p}, G_{\beta i}, G_{5 i}, G_{6 i}, G_{\alpha \kappa}, G_{\kappa \delta}, G_{\delta \alpha}\right\}$ (connected forbidden URLP graphs) in Fig. $3(\mathrm{~m})-(\mathrm{y})$, and $\mathcal{F}_{\text {URLP }}:=\mathcal{T}_{\text {URLP }} \cup \mathcal{D}_{\text {URLP }} \cup \mathcal{C}_{\text {URLP }} 1$.

The labelings in Fig. 9 of $\mathcal{F}_{\text {ULP }}$ were shown to be level non-planar in 910 . In this section, we prove that of these, only the two trees, $T_{8}$ and $T_{9}$, in $\mathcal{T}_{\text {URLP }}$ are also radial level non-planar. We show how to add an extra edge to each of

\footnotetext{
${ }^{1}$ Subscripts $\ell, p$, and $i$ stand for "lone", "pendant", and "internal" edges, respectively.
} 


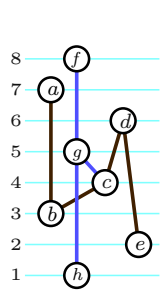

(a) $T_{8}$

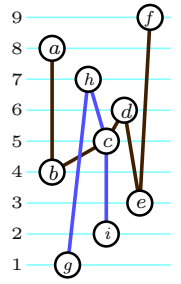

(b) $T_{9}$

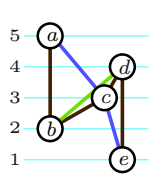

(c) $G_{5}$

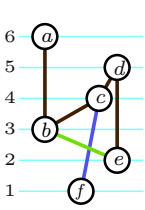

(d) $G_{6}$

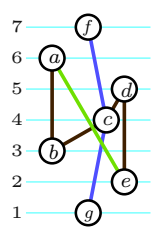

(e) $G_{\alpha}$

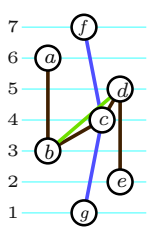

(f) $G_{\kappa}$

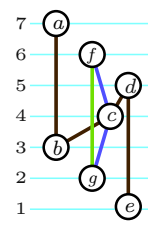

(g) $G_{\delta}$

Fig. 9. Level non-planar labelings of the seven forbidden ULP graphs

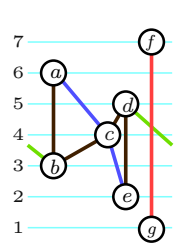

(a) $G_{5} \ell$

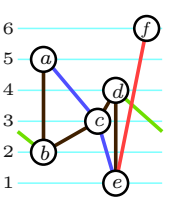

(g) $G_{5 p}$

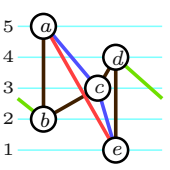

(m) $G_{5 i}$

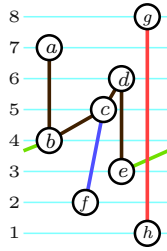

(b) $G_{6 \ell}$

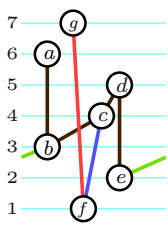

(h) $G_{6 p}$

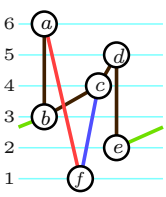

(n) $G_{6 i}$

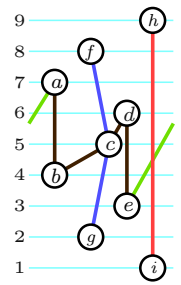

(c) $G_{\alpha \ell}$

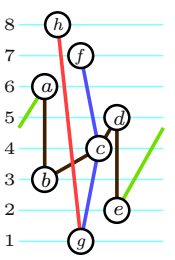

(i) $G_{\alpha p}$

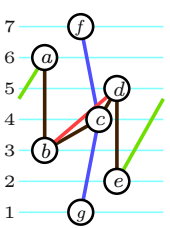

(o) $G_{\alpha}$

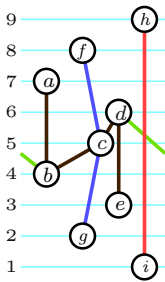

(d) $G_{\kappa \ell}$

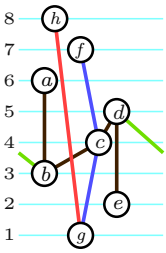

(j) $G_{\kappa p}$

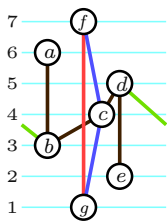

(p) $G_{\kappa \delta}$

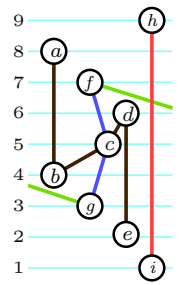

(e) $G_{\delta \ell}$

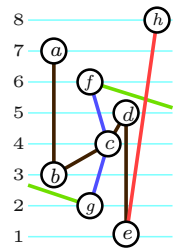

(k) $G_{\delta p}$

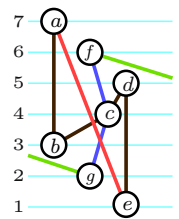

(q) $G_{\delta \alpha}$

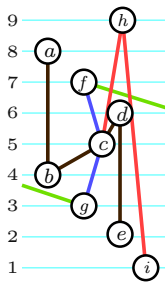

(f) $G_{9}$

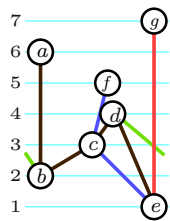

(I) $G_{7}$

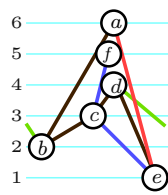

(r) $G_{\beta} i$

Fig. 10. Radial level non-planar labelings of the 20 cyclic forbidden URLP graphs

the five cyclic graphs of $\mathcal{F}_{\text {ULP }}$ in three different ways so as to produce 15 of the graphs of $\mathcal{F}_{\text {URLP }}$ in Fig. $3(\mathrm{~h})-(\mathrm{l})$, (o)-(s), and $(\mathrm{u})-(\mathrm{y})$ that have radial level non-planar labelings in Fig. 10(a)-(e), (g)-(k), and $(\mathrm{m})-(\mathrm{q})$, respectively. For the three remaining graphs of $\mathcal{F}_{\text {URLP }}$ in Fig. $3(\mathrm{~m}),(\mathrm{n})$, and $(\mathrm{t})$, we show that the labelings in Fig. 10(f),(l), and (r), respectively, are also radial level non-planar.

Full proofs for omitted or shortened proofs in this section can be found in 8 . First, we observe a property common to all the embeddings in Figs. 9 and 10.

Observation 6. Suppose a radial level graph $G$ has a labeling $\phi$ and a path $a-b-c-d-e$ such that $\phi(a)>\phi(d)>\phi(c)>\phi(b)>\phi(e)$. For $G$ to be radial level 
planar, $G$ must have an embedding where the path does not wrap such that the edge $a-b$ intersects the tracks $\ell_{c}$ and $\ell_{d}$ to the left of $c$ and $d$ (with respect to the ray that cuts the cylinder as in Fig. Q(b)-(c)), respectively, and the edge $d-e$ intersects the tracks $\ell_{b}$ and $\ell_{c}$ to the right of $b$ and $c$, respectively.

We next see that only $T_{8}$ and $T_{9}$ of $\mathcal{F}_{\text {ULP }}$ are also radial level non-planar.

Lemma 7. Of the seven forbidden ULP graphs in $\mathcal{F}_{U L P}$, only $T_{8}$ and $T_{9}$ are also radial level non-planar with distinct labels.

Proof Sketch: Using the labeling in Fig. 9(a) for $T_{8}$, Observation 6 implies that $a-b-c-d-e$ proceeds left to right. Since the vertex $c$ lies between the edges $a-b$ and $d-e$, so must the edge $c-g$. As a result, either the edge $f-g$ or $g-h$ must cross some edge of $a-b-c-d-e$. A similar argument can be given for $T_{9}$ using the labeling in Fig. 9(b). Finally, each remaining graph is either a generalized circular caterpillar or a tri- $K_{3}$ star, which are URLP by Lemmas 1 and 4

Next we show that all of the graphs in $\mathcal{F}_{\text {URLP }}$ are radial level non-planar.

Lemma 8. There exist distinct labelings preventing each of the cyclic forbidden URLP graphs in $\mathcal{F}_{\text {URLP }}$ from being radial level planar.

Proof Sketch: Each of the 16 graphs in Fig. 10(a)-(k), (m)-(q) contain a subgraph of one of the five cyclic forbidden graphs of $\mathcal{F}_{\text {ULP }}$ using the same relative labeling as in Fig. 9(c)-(g). This means that in order for any of these graphs to be radial level planar, an edge $e$ must wrap. However, in each case (except for $G_{\alpha \kappa}$ ), the graph has an extra edge with extreme labels that prevents $e$ from wrapping. In the case of $G_{\alpha \kappa}$ in Fig. 10(o), if the edge $a-e$ wraps, then the extra edge $b-d$ must cross the path $f-c-g$ whose endpoints have extreme labels. In the cases of $G_{7}$ and $G_{\beta i}$ in Fig. 10(l) and (r), the labeling is the same as the labelings of $G_{\alpha p}$ and $G_{\delta \alpha}$ in Fig. 10(k) and (q) where vertices $e$ and $g$ have been merged into vertex $e$ with an extreme label that forces crossing in each case.

We can extend any radial level non-planar labeling with the next lemma.

Lemma 9. If a graph $G$ contains a subgraph homeomorphic to a graph $\tilde{G}$ with a radial level non-planar labeling, then $G$ also has a radial level non-planar labeling.

Lemma 9 allows us to generalize Lemmas 7 and 8 to the following lemma:

Lemma 10. If a graph contains a subgraph homeomorphic to any of the graphs in $\mathcal{F}_{\text {URLP }}$, then it cannot be URLP with distinct labels.

\section{Characterizing Unlabeled Radial Level Planar Graphs}

In this section, we characterize disconnected and connected URLP graphs separately. The omitted proofs in this section can be found in [8]. 


\subsection{Disconnected Unlabeled Radial Level Planar Graphs}

We characterize the class of disconnected URLP graphs in terms of the forbidden graphs $\mathcal{T}_{\text {URLP }} \cup \mathcal{D}_{\text {URLP }}$. First, we consider minimality.

Lemma 11. Each forbidden graph in $\mathcal{T}_{\text {URLP }}$ and $\mathcal{D}_{\text {URLP }}$ is minimal in that the removal of any edge yields one or more URLP graphs.

In a disconnected graph $G$, each component (discounting isolated vertices) contains at least one edge. Since one of the components of each graph in $\mathcal{D}_{\text {URLP }}$ is an isolated edge, that edge prevents any other component of $G$ from having a subdivision of a cyclic graph from $\mathcal{F}_{\text {ULP }}$. Hence, we can extend Theorem 8.3.13 of [9] that characterizes ULP graphs in terms of $\mathcal{F}_{\text {ULP }}$ to the following theorem: Theorem 12. For a disconnected graph $G$, with no isolated vertices, the following three statements are equivalent:

1. $G$ does not contain a subgraph homeomorphic to $T_{8}, T_{9}, G_{5 \ell}, G_{6 \ell}, G_{\alpha \ell}$, $G_{\kappa \ell}$, or $G_{\delta \ell}$.

2. Each component of $G$ is either a generalized caterpillar, a radius-2 star, an extended 3-spider, or an extended $K_{4}$ subgraph.

3. $G$ is URLP with distinct labels.

\subsection{Connected Unlabeled Radial Level Planar Graphs}

Here we characterize the class of connected URLP graphs in terms of the forbidden graphs $\mathcal{T}_{\text {URLP }} \cup \mathcal{C}_{\text {URLP. }}$. We start by considering minimality.

Lemma 13. Each connected forbidden graph in $\mathcal{C}_{\text {URLP }}$ is minimal in that the removal of any edge that does not disconnect the graph yields an URLP graph.

Lemma 8.3.7 of 9] characterizes generalized caterpillars in terms of the four forbidden graphs in Fig. 11(a)-(d) as follows:

Lemma 14. A connected graph $G$ is a generalized caterpillar if and only if $G$ does not have a subgraph homeomorphic to $G_{6}, C_{5}, G_{\omega}$, or $T_{7}$.

This requires first characterizing graphs in which the maximum length of any cycle is 4, which is Corollary 8.3.2 from [9]:

Lemma 15. Every block of a connected graph $G$ is isomorphic to a $K_{4},\left(K_{3}\right)^{*}$, or $\left(C_{4}\right)^{+}$block, or $G$ contains a $C_{5}$ subdivision.

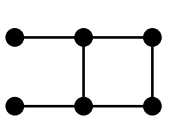

(a) $G_{6}$

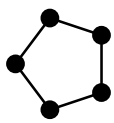

(b) $C_{5}$

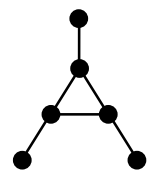

(c) $G_{\omega}$

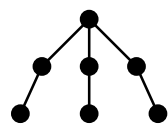

(d) $T_{7}$

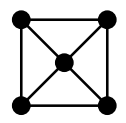

(e) $G_{5 i}$

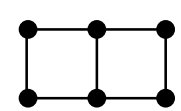

(f) $G_{6}$

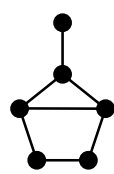

(g) $G_{\beta}$

Fig. 11. Forbidden graphs for generalized caterpillars in (a)-(d) and for generalized circular caterpillars in (d)-(g) 
We extend this characterization to include generalized circular caterpillars in terms of the four forbidden graphs in Fig. 11(d)-(g) as follows:

Lemma 16. A connected graph $G$ is either a generalized caterpillar or a generalized circular caterpillar if and only if $G$ does not contain a subgraph homeomorphic to $T_{7}, G_{5}, G_{6 i}$, or $G_{\beta}$.

Two of the four forbidden graphs of generalized (circular) caterpillars are contained in $\mathcal{C}_{\text {URLP. }}$. For the other two graphs $T_{7}$ and $G_{\beta}$, Figs. 12 and 13 consider all possible ways to add an edge to either obtain an URLP graph or another forbidden graph in $\mathcal{T}_{\text {URLP }} \cup \mathcal{C}_{\text {URLP }}$. This gives the following two lemmas:

Lemma 17. If $G$ is a connected graph that contains a subgraph homeomorphic to $T_{7}$, but does not contain a subgraph homeomorphic to $G_{6 p}, G_{\alpha p}, G_{\kappa p}, G_{\delta p}$, $G_{6 i}, G_{\kappa \delta}, G_{\delta \alpha}, G_{7}, G_{9}$, or $T_{8}$, then $G$ is an extended 3 -spider, a radius-2 star, an tri- $K_{3}$ star, or is isomorphic to $G_{8}$.

Lemma 18. If $G$ is a connected graph that contains a subgraph homeomorphic to $G_{\beta}$, but does not contain a subgraph homeomorphic to $G_{5 p}, G_{6 p}, G_{6 i}, G_{\alpha \kappa}$, $G_{\beta}$, or $T_{8}$, then $G$ is an extended 3-spider.

We next show that $\mathcal{T}_{\text {URLP }} \cup \mathcal{C}_{\text {URLP }}$ forms a set of forbidden URLP graphs with the subsequent lemma; the full proof of which can be found in [8].

Lemma 19. The class of connected URLP graphs with distinct labels does not contain a subgraph homeomorphic to any of graph in $\mathcal{T}_{\text {URLP }} \cup \mathcal{C}_{\text {URLP }}$.

Proof Sketch: By applying Lemma 16] and comparing degree sequences, cycle lengths, and the number of cycles of each graph $G \in \mathcal{T}_{\text {URLP }} \cup \mathcal{C}_{\text {URLP }}$ to each class of URLP graphs, we see that in each case $G$ is a forbidden graph.

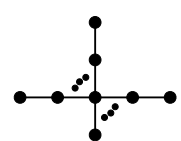

(a) R2S

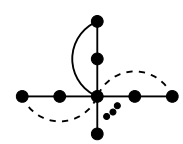

(b) TK3S

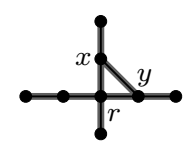

(c) has $G_{\kappa p}$

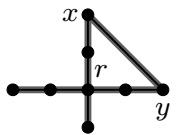

(d) has $G_{\alpha p}$

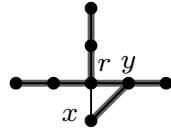

(e) has $T_{8}$

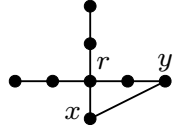

(f) is $G_{8}$

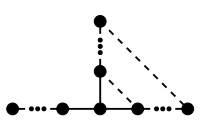

(g) E3S

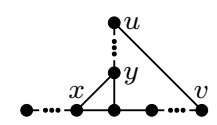

(h) is a E3S

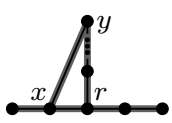

(i) has $G_{6 p}$

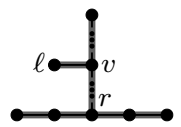

(j) has $T_{8}$

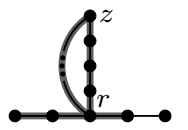

(k) has $G_{6 p}$

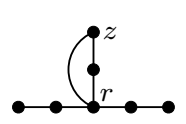

(I) is $G_{\delta}$

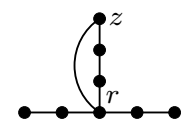

(m) is $G_{8}$

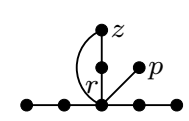

(n) is a TK3S

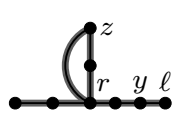

(o) has $G_{\delta p}$

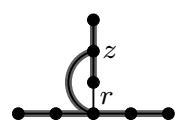

(p) has $T_{8}$

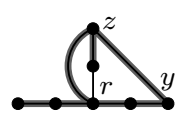

(r) has $G_{6} p$

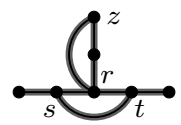

(t) has $G_{\kappa \delta}$

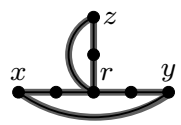

(u) has $G_{\delta \alpha}$

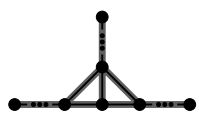

(v) has $G_{7}$

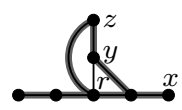

(q) has $G_{6 p}$

Fig. 12. Cases for adding one or more edges to $T_{7}$

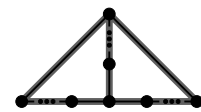

(w) $G_{6}$ 


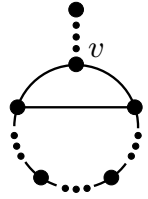

(a) E3S

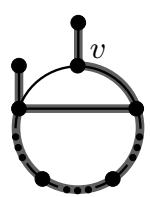

(b) has $G_{6 p}$

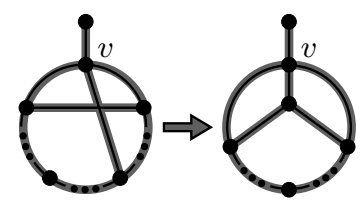

(g) has $G_{\beta}$ i

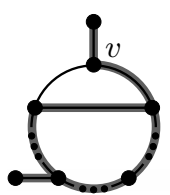

(c) has $G_{6 p}$

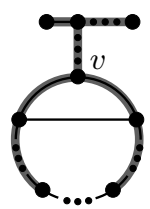

(h) has $T_{8}$

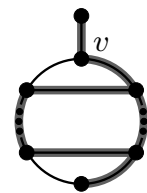

(d) has $G_{6 p}$

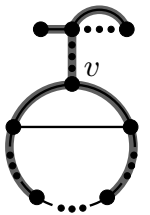

(i) has $T_{8}$

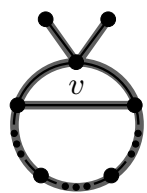

(e) has $G_{\alpha \kappa}$

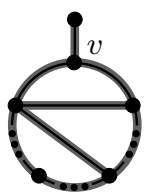

(f) has $G_{5 p}$

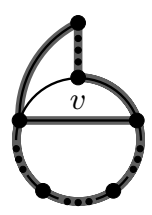

(j) has $G_{6} i$

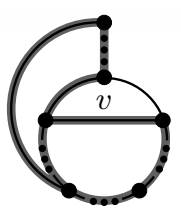

(k) has $G_{6} i$

Fig. 13. Cases for adding one or more edges to $G_{\beta}$

Lemma 20. If $G$ does not contain a subgraph homeomorphic to a graph in $\mathcal{T}_{\text {URLP }} \cup \mathcal{C}_{\text {URLP }}$, then $G$ is a generalized (circular) caterpillar, a radius-2 star, an extended 3-spider, a tri- $K_{3}$ star, or is isomorphic to $G_{8}$.

Proof. If $G$ is not a generalized (circular) caterpillar, then by Lemma $14 G$ must contains a subgraph homeomorphic to $T_{7}, G_{5}, G_{6 i}$, or $G_{\beta}$. However, since both $G_{5 i}$ and $G_{6 i}$ are in $\mathcal{C}_{\text {URLP }}, G$ must either contain a subgraph homeomorphic to $T_{7}$ or $G_{\beta}$. In the first case, $G$ must be an extended 3 -spider, a radius-2 star, tri- $K_{3}$ star, or is isomorphic to $G_{8}$ by Lemma [17. In the second case, $G$ can only be a extended 3-spider by Lemma 18.

Combining Lemmas 1, 2, 3, 4, 5, 10, 19, and 20 gives our final theorem.

Theorem 21. For a connected graph $G$, the following statements are equivalent:

1. $G$ does not contain a subgraph homeomorphic to $T_{8}, T_{9}, G_{7}, G_{9}, G_{\beta} i, G_{5 p}$, $G_{6 p}, G_{\alpha p}, G_{\kappa p}, G_{\delta p}, G_{5 i}, G_{6 i}, G_{\alpha \kappa}, G_{\kappa \delta}$, or $G_{\delta \alpha}$.

2. $G$ is either a generalized caterpillar, a generalized circular caterpillar, a radius-2 star, an extended 3-spider, a tri-K $K_{3}$ star, or $G$ is isomorphic to $G_{8}$.

3. $G$ is URLP with distinct labels.

\section{Conclusion and Future Work}

In this extended abstract, we generalized the ULP characterization to radial level graphs with URLP graphs that are radial level planar for any distinct labeling. We provided separate characterizations for disconnected and connected URLP graphs in terms of 7 and 15 forbidden subdivisions, respectively, along with linear-time drawing algorithms. Future work includes considering the case of duplicate labels and providing linear-time recognition algorithms to determine whether a graph is URLP. 


\section{References}

1. Bachmaier, C., Brandenburg, F.J., Forster, M.: Radial level planarity testing and embedding in linear time. Journal of Graph Algorithms and Applications 9(1), 53-97 (2005)

2. Brandes, U., Kenis, P., Wagner, D.: Communicating centrality in policy network drawings. IEEE Transactions on Visualization and Computer Graphics 09(2), 241253 (2003)

3. Brass, P., Cenek, E., Duncan, C.A., Efrat, A., Erten, C., Ismailescu, D., Kobourov, S.G., Lubiw, A., Mitchell, J.S.B.: On simultaneous graph embedding. Computational Geometry: Theory and Applications 36(2), 117-130 (2007)

4. Cappos, J., Estrella-Balderrama, A., Fowler, J.J., Kobourov, S.G.: Simultaneous graph embedding with bends and circular arcs. Computational Geometry: Theory and Applications 42(2), 173-182 (2009)

5. Eades, P., Feng, Q., Lin, X., Nagamochi, H.: Straight-line drawing algorithms for hierarchical graphs and clustered graphs. Algorithmica 44(1), 1-32 (2006)

6. Estrella-Balderrama, A., Fowler, J.J., Kobourov, S.G.: Characterization of unlabeled level planar trees. In: Kaufmann, M., Wagner, D. (eds.) GD 2006. LNCS, vol. 4372, pp. 367-379. Springer, Heidelberg (2007)

7. Estrella-Balderrama, A., Fowler, J.J., Kobourov, S.G.: Characterization of unlabeled level planar trees. Computational Geometry: Theory and Applications 42(7), 704-721 (2009)

8. Fowler, J.J.: Characterization of unlabeled radial level planar graphs. Technical Report TR09-03, University of Arizona (2009), http://ulp.cs.arizona.edu/TR09-03.pdf

9. Fowler, J.J.: Unlabeled Level Planarity. PhD thesis, University of Arizona (2009), http://ulp.cs.arizona.edu/ulp-thesis.pdf

10. Fowler, J.J., Kobourov, S.G.: Characterization of unlabeled planar graphs. In: Hong, S.-H., Nishizeki, T., Quan, W. (eds.) GD 2007. LNCS, vol. 4875, pp. 37-49. Springer, Heidelberg (2008)

11. Jünger, M., Leipert, S.: Level planar embedding in linear time. Journal of Graph Algorithms and Applications 6(1), 67-113 (2002) 